Acta Crystallographica Section E

\section{Structure Reports}

Online

ISSN 1600-5368

William T. A. Harrison, ${ }^{\text {a }}{ }^{\text {H. S. }}$ Yathirajan, ${ }^{b}$ B. V. Ashalatha, ${ }^{c}$

K. K. Vijaya Raj ${ }^{\mathrm{C}}$ and B. Narayanac

${ }^{\mathrm{a}}$ Department of Chemistry, University of Aberdeen, Meston Walk, Aberdeen AB24 3UE, Scotland, ' ${ }^{\mathbf{D}}$ Department of Studies in Chemistry, University of Mysore, Manasagangotri, Mysore 570 006, India, and ' Department of Chemistry, Mangalore University, Mangalagangotri 574 199, India

Correspondence e-mail: w.harrison@abdn.ac.uk

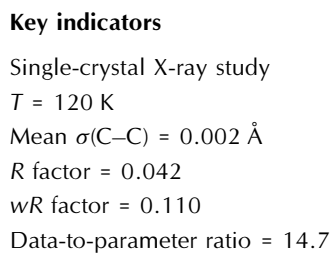

For details of how these key indicators were automatically derived from the article, see http://journals.iucr.org/e.

\title{
Methyl 5-fluoro-1H-indole-2-carboxylate
}

The geometrical parameters for the title compound, $\mathrm{C}_{10} \mathrm{H}_{8} \mathrm{FNO}_{2}$, are normal. In the crystal structure, the molecules form inversion-symmetry-generated dimeric pairs by way of two $\mathrm{N}-\mathrm{H} \cdots \mathrm{O}$ hydrogen bonds.

\section{Comment}

Several indolecarboxylic acid derivatives show biological activity: methyl indole-3-carboxylate, extracted from a marine microorganism (Hu et al., 2005), is cytotoxic against the K562 human leukaemia strain. Methyl indole-2-carboxylic acid may serve as a glycine site antagonist and hence aid in the treatment of human brain injuries (Morzyk-Ociepa et al., 2004). 5Fluoroindole-3-acetic acid (Antolic et al., 1996) has plantgrowth regulating activity. The crystal structure of methyl indole-2-carboxylate has been deposited [Parsons, S., McNab, H. \& Wood, P. (2004). refcode OCAQEP] with the Cambridge Structural Database (CSD; Version 5.27; Allen, 2002). As part of our ongoing research in this area, the structure of the related title compound, (I) (Fig. 1), prepared by the Fischer indole synthesis reaction (Narayana et al., 2005), is now presented.

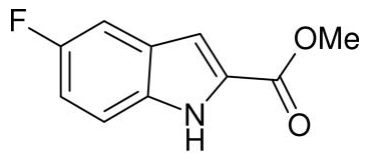

(I)

The geometrical parameters for (I) are consistent with those of the compounds noted above. In particular, methyl indole-2carboxylic acid, (II) (Morzyk-Ociepa et al., 2004), has almost identical geometry to (I). For example, the benzene-ring bond lengths $(\AA)$ in (I) are $\mathrm{C} 1-\mathrm{C} 2=1.396$ (2) [equivalent value in $(\mathrm{II})=1.390$ (2) $\AA$ ], $\mathrm{C} 2-\mathrm{C} 3=1.375$ (2) $[1.372$ (2) $], \mathrm{C} 3-\mathrm{C} 4=$ 1.399 (2) $[1.404$ (2)], $\mathrm{C} 4-\mathrm{C} 5=1.356$ (2) [1.357 (2)], C5-C6 = 1.408 (2) $[1.409$ (2)] and C6-C1 = 1.416 (2) [1.403 (2)]. Apart from the methyl $\mathrm{H}$ atoms, the molecule in (I) is essentially planar [r.m.s. deviation of the non-H atoms from the mean plane $=0.031 \AA$, max. $=0.0327$ (11) $\AA$ for N1]. The bond angle sum about $\mathrm{N} 1$ is $359.7^{\circ}$. The crystal packing in (I) exhibits inversion-symmetry-generated dimeric pairs of molecules linked by two $\mathrm{N}-\mathrm{H}$. . O hydrogen bonds (Table 1 and Fig. 2). A similar pairing arrangement was seen in the structure of methyl indole-2-carboxylate (CSD refcode OCAQEP) although the overall structure is different to (I). Conversely, in methyl indole-2-carboxylic acid (Morzyk-Ociepa et al., 2004) a completely different arrangement of $\mathrm{N}-\mathrm{H} \cdots \mathrm{O}$ and $\mathrm{O}-$ $\mathrm{H}$. O O hydrogen bonds leads to chains of molecules. There are no $\pi-\pi$ stacking interactions in (I), the shortest intermolecular ring-centroid separation being $4.35 \AA$.
Received 8 August 2006 Accepted 9 August 2006
(C) 2006 International Union of Crystallography All rights reserved 


\section{Experimental}

Methyl pyruvate-4-fluorophenylhydrazone (2 g, $0.0095 \mathrm{~mol})$ was added to $10 \mathrm{~g}$ polyphosphoric acid and continuously stirred for proper mixing. The reaction mass was slowly heated to $353-363 \mathrm{~K}$ and maintained for $4 \mathrm{~h}$. The progress of the reaction was monitored by TLC. The reaction mass was cooled and water $(100 \mathrm{ml})$ was added to break up the lumps until it became a slurry. The separated solid was filtered off and washed with water. The dried crude product was charcoalized in ethyl acetate, filtered over hyflo/silica gel, slowly cooled to room temperature and kept overnight with stirring. After recrystallization from ethyl acetate, colourless crystals of (I) were obtained in $60 \%$ yield (m.p. $474 \mathrm{~K}$ ). Analysis found (calculated) for $\mathrm{C}_{10} \mathrm{H}_{8} \mathrm{FNO}_{2}$ : C 62.11 (62.18), H 4.09 (4.17), N 7.13 (7.25)\%.

\section{Crystal data}

$\mathrm{C}_{10} \mathrm{H}_{8} \mathrm{FNO}_{2}$

$M_{r}=193.17$

Monoclinic, $P 2_{1} / n$

$a=12.4420(7) \AA$

$b=3.8185$ (1) ̊

$c=18.269(1) \AA$

$\beta=100.125(2)^{\circ}$

$V=854.43(7) \AA^{3}$

$$
\begin{aligned}
& Z=4 \\
& D_{x}=1.502 \mathrm{Mg} \mathrm{m}^{-3} \\
& \text { Mo } K \alpha \text { radiation } \\
& \mu=0.12 \mathrm{~mm}^{-1} \\
& T=120(2) \mathrm{K} \\
& \text { Needle, colourless } \\
& 0.41 \times 0.07 \times 0.05 \mathrm{~mm}
\end{aligned}
$$

\section{Data collection}

Nonius KappaCCD diffractometer $\varphi$ and $\omega$ scans

Absorption correction: multi-scan (SADABS; Bruker, 2003)

$T_{\min }=0.952, T_{\max }=0.994$

\section{Refinement}

Refinement on $F^{2}$

$R\left[F^{2}>2 \sigma\left(F^{2}\right)\right]=0.042$

$w R\left(F^{2}\right)=0.110$

$S=1.03$

1937 reflections

132 parameters

$\mathrm{H}$ atoms treated by a mixture of independent and constrained refinement

10458 measured reflections 1937 independent reflections 1311 reflections with $I>2 \sigma(I)$ $R_{\text {int }}=0.052$

$\theta_{\max }=27.6^{\circ}$

$$
\begin{aligned}
& w=1 /\left[\sigma^{2}\left(F_{\mathrm{o}}^{2}\right)+(0.0568 P)^{2}\right. \\
& +0.104 P] \\
& \text { where } P=\left(F_{\mathrm{o}}{ }^{2}+2 F_{\mathrm{c}}{ }^{2}\right) / 3 \\
& (\Delta / \sigma)_{\max }<0.001
\end{aligned}
$$

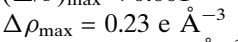

$$
\begin{aligned}
& \Delta \rho_{\min }=-0.26 \mathrm{e}^{-3} \\
& \text { Extinction correction: SHELXL97 } \\
& \text { Extinction coefficient: } 0.014 \text { (3) }
\end{aligned}
$$

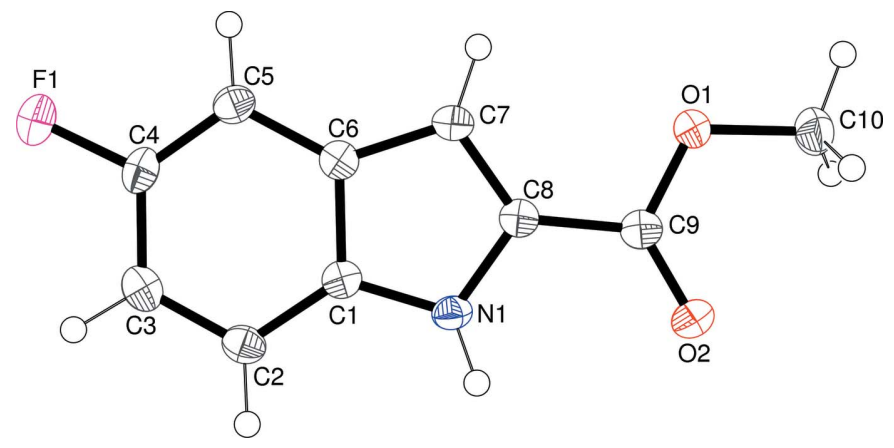

Figure 1

View of (I), showing $50 \%$ probability displacement ellipsoids and arbitrary spheres for the $\mathrm{H}$ atoms.

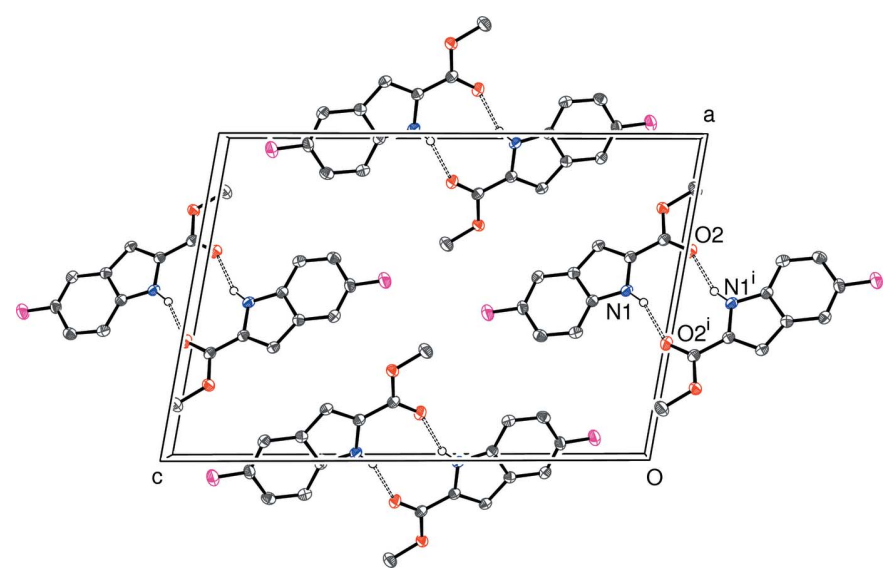

Figure 2

Unit cell packing in (I) with all $\mathrm{H}$ atoms except $\mathrm{H} 1$ omitted for clarity and hydrogen bonds indicated by dashed lines. See Table 1 for symmetry code.

We thank the EPSRC National Crystallography Service (University of Southampton, England) for data collection. ABV thanks Mangalore University for provision of research facilities.

\section{References}

Allen, F. H. (2002). Acta Cryst. B58, 380-388

Antolić, A., Kojić-Prodić, B., Tomić, S., Nigović, B., Magnus, V. \& Cohen, J. D. (1996). Acta Cryst. B52, 651-661.

Blessing, R. H. (1995). Acta Cryst. A51, 33-38.

Bruker (2003). SADABS. Version 2.10. Bruker AXS Inc., Madison, Wisconsin, USA.

Farrugia, L. J. (1997). J. Appl. Cryst. 30, 565.

Hu, S.-C., Tan, R.-X., Hong, K., Yu, Z.-N. \& Zhu, H.-L. (2005). Acta Cryst. E61, o1654-o1656.

Morzyk-Ociepa, B., Michalska, D. \& Pietraszko, A. (2004). J. Mol. Struct. 688, 79-86.

Narayana, B., Ashalatha, B. V., Vijaya Raj, K. K., Fernandes, J. \& Sarojini, B. K. (2005). Bioorg. Med. Chem. 13, 4638-4644.

Nonius (1998). COLLECT. Nonius BV, Delft, The Netherlands.

Otwinowski, Z. \& Minor, W. (1997). Methods in Enzymology, Vol. 276, Macromolecular Crystallography, Part A, edited by C. W. Carter Jr \& R. M. Sweet, pp. 307-326. New York: Academic Press.

Sheldrick, G. M. (1997). SHELXS97 and SHELXL97. University of Göttingen, Germany. 


\section{supporting information}

Acta Cryst. (2006). E62, o4050-o4051 [https://doi.org/10.1107/S1600536806031448]

\section{Methyl 5-fluoro-1H-indole-2-carboxylate}

William T. A. Harrison, H. S. Yathirajan, B. V. Ashalatha, K. K. Vijaya Raj and B. Narayana

Methyl 5-fluoro-1H-indole-2-carboxylate

Crystal data

$\mathrm{C}_{10} \mathrm{H}_{8} \mathrm{FNO}_{2}$

$M_{r}=193.17$

Monoclinic, $P 2_{1} / n$

Hall symbol: -P 2 yn

$a=12.4420(7) \AA$

$b=3.8185(1) \AA$

$c=18.269(1) \AA$

$\beta=100.125(2)^{\circ}$

$V=854.43(7) \AA^{3}$

$Z=4$

\section{Data collection}

Nonius KappaCCD diffractometer

Radiation source: fine-focus sealed tube Graphite monochromator $\varphi$ and $\omega$ scans

Absorption correction: multi-scan

(SADABS; Bruker, 2003)

$T_{\min }=0.952, T_{\max }=0.994$

\section{Refinement}

Refinement on $F^{2}$

Least-squares matrix: full

$R\left[F^{2}>2 \sigma\left(F^{2}\right)\right]=0.042$

$w R\left(F^{2}\right)=0.110$

$S=1.03$

1937 reflections

132 parameters

0 restraints

Primary atom site location: structure-invariant direct methods

Secondary atom site location: difference Fourier map
$F(000)=400$

$D_{\mathrm{x}}=1.502 \mathrm{Mg} \mathrm{m}^{-3}$

Mo $K \alpha$ radiation, $\lambda=0.71073 \AA$

Cell parameters from 2189 reflections

$\theta=1.0-27.5^{\circ}$

$\mu=0.12 \mathrm{~mm}^{-1}$

$T=120 \mathrm{~K}$

Needle, colourless

$0.41 \times 0.07 \times 0.05 \mathrm{~mm}$

10458 measured reflections

1937 independent reflections

1311 reflections with $I>2 \sigma(I)$

$R_{\text {int }}=0.052$

$\theta_{\max }=27.6^{\circ}, \theta_{\min }=4.0^{\circ}$

$h=-16 \rightarrow 16$

$k=-4 \rightarrow 4$

$l=-22 \rightarrow 23$

Hydrogen site location: difmap and geom

$\mathrm{H}$ atoms treated by a mixture of independent and constrained refinement

$w=1 /\left[\sigma^{2}\left(F_{\mathrm{o}}^{2}\right)+(0.0568 P)^{2}+0.104 P\right]$

where $P=\left(F_{\mathrm{o}}{ }^{2}+2 F_{\mathrm{c}}{ }^{2}\right) / 3$

$(\Delta / \sigma)_{\max }<0.001$

$\Delta \rho_{\max }=0.23 \mathrm{e} \AA^{-3}$

$\Delta \rho_{\min }=-0.26$ e $\AA^{-3}$

Extinction correction: SHELXL97, $\mathrm{Fc}^{*}=\mathrm{kFc}\left[1+0.001 \mathrm{xFc}^{2} \lambda^{3} / \sin (2 \theta)\right]^{-1 / 4}$

Extinction coefficient: 0.014 (3)

\section{Special details}

Experimental. 1H NMR (CDCl3, 300?MHz): $\delta 3.92(\mathrm{~s}, 3 \mathrm{H},-\mathrm{CH} 3), 7.01(\mathrm{dt}, 1 \mathrm{H}, \mathrm{Ar}-\mathrm{H}), 7.10(\mathrm{~s}, 1 \mathrm{H}, \mathrm{Ar}-\mathrm{H}), 7.25(\mathrm{dd}$, $\mathrm{J}=2.4$ and $9.3 \mathrm{~Hz}, 1 \mathrm{H}, \mathrm{Ar}-\mathrm{H}), 7.44(\mathrm{dd}, \mathrm{J}=4.2$ and $8.7 \mathrm{~Hz}, 1 \mathrm{H}, \mathrm{Ar}-\mathrm{H}), 11.38$ (br s, $1 \mathrm{H},-\mathrm{NH}-$, exchangeable with $\mathrm{D} 2 \mathrm{O})$. 
Geometry. All e.s.d.'s (except the e.s.d. in the dihedral angle between two 1.s. planes) are estimated using the full covariance matrix. The cell e.s.d.'s are taken into account individually in the estimation of e.s.d.'s in distances, angles and torsion angles; correlations between e.s.d.'s in cell parameters are only used when they are defined by crystal symmetry. An approximate (isotropic) treatment of cell e.s.d.'s is used for estimating e.s.d.'s involving 1.s. planes.

Refinement. Refinement of $F^{2}$ against ALL reflections. The weighted $R$-factor $w R$ and goodness of fit $S$ are based on $F^{2}$, conventional $R$-factors $R$ are based on $F$, with $F$ set to zero for negative $F^{2}$. The threshold expression of $F^{2}>\sigma\left(F^{2}\right)$ is used only for calculating $R$-factors(gt) $e t c$. and is not relevant to the choice of reflections for refinement. $R$-factors based on $F^{2}$ are statistically about twice as large as those based on $F$, and $R$ - factors based on ALL data will be even larger.

Fractional atomic coordinates and isotropic or equivalent isotropic displacement parameters $\left(\AA^{2}\right)$

\begin{tabular}{|c|c|c|c|c|}
\hline & $x$ & $y$ & $z$ & $U_{\text {iso }} * / U_{\text {eq }}$ \\
\hline $\mathrm{C} 1$ & $0.48721(12)$ & 0.2695 (4) & $0.17446(9)$ & $0.0207(4)$ \\
\hline $\mathrm{C} 2$ & $0.39008(13)$ & $0.1753(4)$ & $0.19763(9)$ & 0.0241 (4) \\
\hline $\mathrm{H} 2$ & 0.3330 & 0.0610 & 0.1649 & $0.029 *$ \\
\hline $\mathrm{C} 3$ & $0.38010(13)$ & $0.2540(4)$ & $0.26959(9)$ & $0.0255(4)$ \\
\hline H3 & 0.3156 & 0.1922 & 0.2877 & $0.031 *$ \\
\hline $\mathrm{C} 4$ & $0.46546(14)$ & $0.4259(4)$ & $0.31588(9)$ & $0.0252(4)$ \\
\hline $\mathrm{C} 5$ & $0.56073(13)$ & $0.5227(4)$ & $0.29536(9)$ & $0.0232(4)$ \\
\hline H5 & 0.6165 & 0.6391 & 0.3288 & $0.028 *$ \\
\hline C6 & $0.57298(13)$ & $0.4421(4)$ & $0.22209(9)$ & $0.0204(4)$ \\
\hline $\mathrm{C} 7$ & $0.65781(12)$ & 0.4953 (4) & $0.18042(9)$ & $0.0210(4)$ \\
\hline $\mathrm{H} 7$ & 0.7259 & 0.6068 & 0.1972 & $0.025 *$ \\
\hline $\mathrm{C} 8$ & $0.62237(12)$ & $0.3543(4)$ & $0.11111(9)$ & $0.0200(4)$ \\
\hline $\mathrm{C} 9$ & $0.67713(12)$ & $0.3284(4)$ & $0.04739(9)$ & $0.0216(4)$ \\
\hline $\mathrm{C} 10$ & 0.83499 (14) & $0.4799(5)$ & $-0.00077(10)$ & $0.0318(4)$ \\
\hline $\mathrm{H} 10 \mathrm{~A}$ & 0.9051 & 0.5999 & 0.0142 & $0.048^{*}$ \\
\hline $\mathrm{H} 10 \mathrm{~B}$ & 0.7929 & 0.5979 & -0.0442 & $0.048^{*}$ \\
\hline $\mathrm{H} 10 \mathrm{C}$ & 0.8481 & 0.2360 & -0.0134 & $0.048^{*}$ \\
\hline N1 & $0.51896(10)$ & $0.2191(3)$ & $0.10736(8)$ & $0.0213(3)$ \\
\hline $\mathrm{H} 1$ & $0.4827(14)$ & $0.101(4)$ & $0.0694(10)$ & $0.026^{*}$ \\
\hline $\mathrm{O} 1$ & $0.77453(9)$ & $0.4888(3)$ & $0.05982(6)$ & $0.0247(3)$ \\
\hline $\mathrm{O} 2$ & $0.64084(9)$ & $0.1788(3)$ & $-0.01054(6)$ & $0.0286(3)$ \\
\hline F1 & $0.44961(8)$ & $0.5000(3)$ & $0.38680(5)$ & $0.0356(3)$ \\
\hline
\end{tabular}

Atomic displacement parameters $\left(\AA^{2}\right)$

\begin{tabular}{lllllll}
\hline & $U^{11}$ & $U^{22}$ & $U^{33}$ & $U^{12}$ & $U^{13}$ & $U^{23}$ \\
\hline C1 & $0.0213(8)$ & $0.0185(8)$ & $0.0214(9)$ & $0.0024(6)$ & $0.0017(7)$ & $0.0013(6)$ \\
C2 & $0.0209(8)$ & $0.0225(9)$ & $0.0277(10)$ & $-0.0002(7)$ & $0.0013(7)$ & $0.0015(7)$ \\
C3 & $0.0231(9)$ & $0.0231(9)$ & $0.0313(10)$ & $0.0032(7)$ & $0.0076(7)$ & $0.0044(7)$ \\
C4 & $0.0299(9)$ & $0.0267(9)$ & $0.0199(9)$ & $0.0059(7)$ & $0.0071(7)$ & $0.0002(7)$ \\
C5 & $0.0240(9)$ & $0.0229(9)$ & $0.0216(9)$ & $0.0012(6)$ & $0.0007(7)$ & $0.0004(7)$ \\
C6 & $0.0212(8)$ & $0.0168(8)$ & $0.0229(9)$ & $0.0027(6)$ & $0.0030(6)$ & $0.0023(6)$ \\
C7 & $0.0200(8)$ & $0.0189(8)$ & $0.0230(9)$ & $-0.0008(6)$ & $0.0002(7)$ & $0.0007(7)$ \\
C8 & $0.0207(8)$ & $0.0176(8)$ & $0.0210(9)$ & $0.0025(6)$ & $0.0015(6)$ & $0.0031(6)$ \\
C9 & $0.0223(8)$ & $0.0196(8)$ & $0.0216(9)$ & $0.0029(7)$ & $0.0001(7)$ & $0.0037(7)$ \\
C10 & $0.0327(10)$ & $0.0346(10)$ & $0.0310(11)$ & $-0.0048(8)$ & $0.0137(8)$ & $-0.0018(8)$ \\
N1 & $0.0191(7)$ & $0.0225(7)$ & $0.0208(8)$ & $0.0001(5)$ & $-0.0004(5)$ & $-0.0027(6)$
\end{tabular}




\begin{tabular}{lllllll}
$\mathrm{O} 1$ & $0.0231(6)$ & $0.0287(7)$ & $0.0231(7)$ & $-0.0044(5)$ & $0.0064(5)$ & $-0.0024(5)$ \\
$\mathrm{O} 2$ & $0.0289(7)$ & $0.0352(7)$ & $0.0210(7)$ & $-0.0044(5)$ & $0.0022(5)$ & $-0.0051(5)$ \\
$\mathrm{F} 1$ & $0.0394(6)$ & $0.0447(7)$ & $0.0254(6)$ & $-0.0011(5)$ & $0.0127(5)$ & $-0.0044(5)$ \\
\hline
\end{tabular}

Geometric parameters $\left(\stackrel{A}{\circ}{ }^{\circ}\right)$

\begin{tabular}{|c|c|c|c|}
\hline $\mathrm{C} 1-\mathrm{N} 1$ & $1.366(2)$ & $\mathrm{C} 7-\mathrm{C} 8$ & $1.375(2)$ \\
\hline $\mathrm{C} 1-\mathrm{C} 2$ & $1.396(2)$ & $\mathrm{C} 7-\mathrm{H} 7$ & 0.9500 \\
\hline $\mathrm{C} 1-\mathrm{C} 6$ & $1.416(2)$ & $\mathrm{C} 8-\mathrm{N} 1$ & $1.377(2)$ \\
\hline $\mathrm{C} 2-\mathrm{C} 3$ & $1.375(2)$ & $\mathrm{C} 8-\mathrm{C} 9$ & $1.452(2)$ \\
\hline $\mathrm{C} 2-\mathrm{H} 2$ & 0.9500 & $\mathrm{C} 9-\mathrm{O} 2$ & $1.2173(18)$ \\
\hline $\mathrm{C} 3-\mathrm{C} 4$ & $1.399(2)$ & $\mathrm{C} 9-\mathrm{O} 1$ & $1.3412(19)$ \\
\hline $\mathrm{C} 3-\mathrm{H} 3$ & 0.9500 & $\mathrm{C} 10-\mathrm{O} 1$ & $1.444(2)$ \\
\hline $\mathrm{C} 4-\mathrm{C} 5$ & $1.356(2)$ & $\mathrm{C} 10-\mathrm{H} 10 \mathrm{~A}$ & 0.9800 \\
\hline $\mathrm{C} 4-\mathrm{F} 1$ & $1.3739(19)$ & $\mathrm{C} 10-\mathrm{H} 10 \mathrm{~B}$ & 0.9800 \\
\hline $\mathrm{C} 5-\mathrm{C} 6$ & $1.408(2)$ & $\mathrm{C} 10-\mathrm{H} 10 \mathrm{C}$ & 0.9800 \\
\hline $\mathrm{C} 5-\mathrm{H} 5$ & 0.9500 & $\mathrm{~N} 1-\mathrm{H} 1$ & $0.883(18)$ \\
\hline $\mathrm{C} 6-\mathrm{C} 7$ & $1.420(2)$ & & \\
\hline $\mathrm{N} 1-\mathrm{C} 1-\mathrm{C} 2$ & $129.66(14)$ & $\mathrm{C} 8-\mathrm{C} 7-\mathrm{H} 7$ & 126.6 \\
\hline $\mathrm{N} 1-\mathrm{C} 1-\mathrm{C} 6$ & $108.14(14)$ & $\mathrm{C} 6-\mathrm{C} 7-\mathrm{H} 7$ & 126.6 \\
\hline $\mathrm{C} 2-\mathrm{C} 1-\mathrm{C} 6$ & $122.19(15)$ & $\mathrm{C} 7-\mathrm{C} 8-\mathrm{N} 1$ & $109.76(14)$ \\
\hline $\mathrm{C} 3-\mathrm{C} 2-\mathrm{C} 1$ & $117.52(15)$ & $\mathrm{C} 7-\mathrm{C} 8-\mathrm{C} 9$ & $130.19(15)$ \\
\hline $\mathrm{C} 3-\mathrm{C} 2-\mathrm{H} 2$ & 121.2 & $\mathrm{~N} 1-\mathrm{C} 8-\mathrm{C} 9$ & $120.04(14)$ \\
\hline $\mathrm{C} 1-\mathrm{C} 2-\mathrm{H} 2$ & 121.2 & $\mathrm{O} 2-\mathrm{C} 9-\mathrm{O} 1$ & $123.19(15)$ \\
\hline $\mathrm{C} 2-\mathrm{C} 3-\mathrm{C} 4$ & $119.59(15)$ & $\mathrm{O} 2-\mathrm{C} 9-\mathrm{C} 8$ & $125.07(15)$ \\
\hline $\mathrm{C} 2-\mathrm{C} 3-\mathrm{H} 3$ & 120.2 & $\mathrm{O} 1-\mathrm{C} 9-\mathrm{C} 8$ & $111.74(13)$ \\
\hline $\mathrm{C} 4-\mathrm{C} 3-\mathrm{H} 3$ & 120.2 & $\mathrm{O} 1-\mathrm{C} 10-\mathrm{H} 10 \mathrm{~A}$ & 109.5 \\
\hline $\mathrm{C} 5-\mathrm{C} 4-\mathrm{F} 1$ & $118.81(14)$ & $\mathrm{O} 1-\mathrm{C} 10-\mathrm{H} 10 \mathrm{~B}$ & 109.5 \\
\hline $\mathrm{C} 5-\mathrm{C} 4-\mathrm{C} 3$ & $124.63(16)$ & $\mathrm{H} 10 \mathrm{~A}-\mathrm{C} 10-\mathrm{H} 10 \mathrm{~B}$ & 109.5 \\
\hline $\mathrm{F} 1-\mathrm{C} 4-\mathrm{C} 3$ & $116.56(14)$ & $\mathrm{O} 1-\mathrm{C} 10-\mathrm{H} 10 \mathrm{C}$ & 109.5 \\
\hline $\mathrm{C} 4-\mathrm{C} 5-\mathrm{C} 6$ & $116.71(15)$ & $\mathrm{H} 10 \mathrm{~A}-\mathrm{C} 10-\mathrm{H} 10 \mathrm{C}$ & 109.5 \\
\hline $\mathrm{C} 4-\mathrm{C} 5-\mathrm{H} 5$ & 121.6 & $\mathrm{H} 10 \mathrm{~B}-\mathrm{C} 10-\mathrm{H} 10 \mathrm{C}$ & 109.5 \\
\hline $\mathrm{C} 6-\mathrm{C} 5-\mathrm{H} 5$ & 121.6 & $\mathrm{C} 1-\mathrm{N} 1-\mathrm{C} 8$ & $108.52(13)$ \\
\hline $\mathrm{C} 5-\mathrm{C} 6-\mathrm{C} 1$ & $119.36(15)$ & $\mathrm{C} 1-\mathrm{N} 1-\mathrm{H} 1$ & $126.1(11)$ \\
\hline $\mathrm{C} 5-\mathrm{C} 6-\mathrm{C} 7$ & $133.90(15)$ & $\mathrm{C} 8-\mathrm{N} 1-\mathrm{H} 1$ & $125.1(11)$ \\
\hline $\mathrm{C} 1-\mathrm{C} 6-\mathrm{C} 7$ & $106.74(14)$ & $\mathrm{C} 9-\mathrm{O} 1-\mathrm{C} 10$ & $115.88(12)$ \\
\hline $\mathrm{C} 8-\mathrm{C} 7-\mathrm{C} 6$ & $106.83(14)$ & & \\
\hline $\mathrm{N} 1-\mathrm{C} 1-\mathrm{C} 2-\mathrm{C} 3$ & $178.99(15)$ & $\mathrm{C} 1-\mathrm{C} 6-\mathrm{C} 7-\mathrm{C} 8$ & $-0.57(16)$ \\
\hline $\mathrm{C} 6-\mathrm{C} 1-\mathrm{C} 2-\mathrm{C} 3$ & $-0.5(2)$ & $\mathrm{C} 6-\mathrm{C} 7-\mathrm{C} 8-\mathrm{N} 1$ & $0.64(17)$ \\
\hline $\mathrm{C} 1-\mathrm{C} 2-\mathrm{C} 3-\mathrm{C} 4$ & $0.7(2)$ & $\mathrm{C} 6-\mathrm{C} 7-\mathrm{C} 8-\mathrm{C} 9$ & $-178.29(15)$ \\
\hline $\mathrm{C} 2-\mathrm{C} 3-\mathrm{C} 4-\mathrm{C} 5$ & $-0.5(3)$ & $\mathrm{C} 7-\mathrm{C} 8-\mathrm{C} 9-\mathrm{O} 2$ & $175.35(15)$ \\
\hline $\mathrm{C} 2-\mathrm{C} 3-\mathrm{C} 4-\mathrm{F} 1$ & $179.30(13)$ & $\mathrm{N} 1-\mathrm{C} 8-\mathrm{C} 9-\mathrm{O} 2$ & $-3.5(2)$ \\
\hline $\mathrm{F} 1-\mathrm{C} 4-\mathrm{C} 5-\mathrm{C} 6$ & $-179.78(12)$ & $\mathrm{C} 7-\mathrm{C} 8-\mathrm{C} 9-\mathrm{O} 1$ & $-4.2(2)$ \\
\hline $\mathrm{C} 3-\mathrm{C} 4-\mathrm{C} 5-\mathrm{C} 6$ & $0.0(2)$ & $\mathrm{N} 1-\mathrm{C} 8-\mathrm{C} 9-\mathrm{O} 1$ & $176.93(12)$ \\
\hline $\mathrm{C} 4-\mathrm{C} 5-\mathrm{C} 6-\mathrm{C} 1$ & $0.2(2)$ & $\mathrm{C} 2-\mathrm{C} 1-\mathrm{N} 1-\mathrm{C} 8$ & $-179.43(15)$ \\
\hline $\mathrm{C} 4-\mathrm{C} 5-\mathrm{C} 6-\mathrm{C} 7$ & $-179.57(16)$ & $\mathrm{C} 6-\mathrm{C} 1-\mathrm{N} 1-\mathrm{C} 8$ & $0.09(17)$ \\
\hline
\end{tabular}


supporting information

$\begin{array}{llll}\mathrm{N} 1-\mathrm{C} 1-\mathrm{C} 6-\mathrm{C} 5 & -179.56(13) & \mathrm{C} 7-\mathrm{C} 8-\mathrm{N} 1-\mathrm{C} 1 & -0.46(17) \\ \mathrm{C} 2-\mathrm{C} 1-\mathrm{C} 6-\mathrm{C} 5 & 0.0(2) & \mathrm{C} 9-\mathrm{C} 8-\mathrm{N} 1-\mathrm{C} 1 & 178.59(13) \\ \mathrm{N} 1-\mathrm{C} 1-\mathrm{C} 6-\mathrm{C} 7 & 0.30(16) & \mathrm{O} 2-\mathrm{C} 9-\mathrm{O} 1-\mathrm{C} 10 & 1.0(2) \\ \mathrm{C} 2-\mathrm{C} 1-\mathrm{C} 6-\mathrm{C} 7 & 179.86(14) & \mathrm{C} 8-\mathrm{C} 9-\mathrm{O} 1-\mathrm{C} 10 & -179.46(13) \\ \mathrm{C} 5-\mathrm{C} 6-\mathrm{C} 7-\mathrm{C} 8 & 179.26(16) & & \end{array}$

Hydrogen-bond geometry $\left(\AA,{ }^{\circ}\right)$

\begin{tabular}{lllll}
\hline$D-\mathrm{H} \cdots A$ & $D-\mathrm{H}$ & $\mathrm{H} \cdots A$ & $D \cdots A$ & $D-\mathrm{H} \cdots A$ \\
\hline $\mathrm{N} 1-\mathrm{H} 1 \cdots \mathrm{O} 2^{\mathrm{i}}$ & $0.883(18)$ & $2.019(18)$ & $2.8555(18)$ & $157.7(15)$
\end{tabular}

Symmetry code: (i) $-x+1,-y,-z$. 